\title{
Poor Health Literacy, Dilapidated Referral System, Step-Wise Commission Business and Huge Out of Pocket Expense: The Hardcore Challenges of Private Health Services in Bangladesh
}

\author{
S.M. Yasir Arafat* \\ Department of Psychiatry, Enam Medical College and Hospital, Savar, Bangladesh \\ *Corresponding Author: S.M. Yasir Arafat, Department of Psychiatry, Enam Medical College and \\ Hospital, Savar, Bangladesh.Email:arafatdmc62@gmail.com
}

\section{Personal View}

In Bangladesh, majority of people initially visit nearby pharmacy man (they are also called chemist) to consult, instead of visiting the general practitioners or doctors when they feel sick. The chemist gives them medications and follows up them further. When they fail to cure the disease then they refer the patients. These chemists are the prime source of referral to the chamber complexes. The consultation centers visit the chemists/drug sellers and maintain liaison to get patients. In turn they are being given a commission from bill of investigations of the referredpatients. During consultation, consultation center management sends reminder to the consultant that the patient has been referred by a chemist pressuring to give more investigations so that the chemist gets more money which will reinforce the chemist and bolster the further referral. This silent game happens in disguised manner and the patient never understands what is happening to deceive him/her. If any physician practices ethically, doesn't suggest more investigations, the chemist would not refer further patients to him/her. The poor people have been losing their money for the malevolent chemists and disastrous health system. Personally, I think few core issues are responsible for this condition such as poor health literacy, non-existence of referral system, systemized commission business and out of pocket expense on health.

Bangladesh is a densely populated rising economy where literacy rate is rising however, health literacy is very poor $[1,2]$. Instead of searching in appropriate source, visiting registered doctors or general practitioners, people used to visit pharmacy and chemists are taking tolls of the poor health literacy. These hidden burglary costs extra expenses for the poor.

Bangladesh has been achieving health indicators progressively viz achieved few Millennium Development Goals. However, private practice domain is totally unregulated as there is no established referral system [3]. People can, want to, and used to visit professors for simple fever; most professors are consulting such patients with very few referral habits. Some consultants have been consulting more than 100 patients in a day and people are like to visit them because of unknown reasons. Sometimes patients request physicians to give expensive investigations like CT scan of head/MRI of brain. Sometimes, patients comment that the physician is not good if (s) he doesn't suggest any investigation. If there is an entry point in a specified area and an established referral system, role of chemist would abolish resulting in breaking the chemist middleman ship.

Chemists have been paid by the consultation centers/ clinics, doctors are taking referral fee from the diagnostic centers and diagnostic centers have been charging a high rate from the poor patients. This practice cannot be justified by any means. However, it has become an open secret and nobody is taking any initiatives to prevent this in Bangladesh.

Health insurance culture has not been started in Bangladesh and people have to pay for their illness as out of pocket expense which in turn makes them more poor and vulnerable as a consequence of further deterioration in socioeconomic determinants. Sometimes people have to sell their lands, ornaments and other resources to meet the expenses of treatment. 
Malevolent activities starts from the initial moves to chemists where chemists give them the medicines those are more profitable instead of medicines of reputed companies, referring to a physician/consultation center which gives more investigation and more commission from the investigation bill. Second phase starts when physicians have been paid from diagnostic centers from the investigation bill. The issue becomes more complex because of having no referral habit to other specialties. End of the day the people have been exploited by chemist, consultation centers, diagnostic centers and even by the physicians which makes them more poor. Health is a basic right of a citizen however, how could a person can get this right appropriately in such a system. Only ethical practice of the physicians would not solve the issue. Health literacy is the key concern to address, structured referral system could regulate it and legal actions to stop the commission business should take into account.

\section{REFERENCES}

[1] Arafat SMY, Majumder MAA, KabirR, Papadopoulos K, Uddin MS. Health Literacy in School In: Papalois and Theodosopoulou, editor. Optimizing health literacy for improved clinical practices. Hershey, PA: Medical Information Science Reference; 2018; 175-197.

[2] Arafat SMY, Ahmed S. Burden of Misconception in Sexual Health Care Setting: A Cross-Sectional Investigation among the Patients Attending a Psychiatric Sex Clinic of Bangladesh. Psychiatry J. 2017;2017:9827083.

[3] Arafat SMY. Doctor Patient Relationship: an Untouched Issue in Bangladesh. Int $\mathbf{J}$ Psychiatry. 2016; 1(1):2.

Citation: S.M. Yasir Arafat, Poor Health Literacy, Dilapidated Referral System, Step-Wise Commission Business and Huge Out of Pocket Expense: The Hardcore Challenges of Private Health Services in Bangladesh. ARC Journal of Public Health and Community Medicine.2019; 4(3):28-29. DOI: dx.doi.org/ 10.204 31/2456-0596.0403003.

Copyright: (C) 2019 Authors. This is an open-access article distributed under the terms of the Creative Commons Attribution License, which permits unrestricted use, distribution, and reproduction in any medium, provided the original author and source are credited. 